\title{
Therapeutic efficacy of fibroblast growth factor 10 in a rabbit model of dry eye
}

\author{
WENJING ZHENG ${ }^{1}$, MINGMING MA $^{1}$, ERGANG DU ${ }^{2}$, ZHENGWEI ZHANG $^{1}$, \\ KELIMU JIANG ${ }^{1}$, QING GU ${ }^{1}$ and BILIAN KE ${ }^{1}$
}

${ }^{1}$ Department of Ophthalmology, Shanghai General Hospital, Shanghai Jiaotong University School of Medicine, Shanghai 200080; ${ }^{2}$ Department of Ophthalmology, Zhejiang Provincial People's Hospital, Hangzhou, Zhejiang 310014, P.R. China

Received October 2, 2014; Accepted June 26, 2015

DOI: $10.3892 / \mathrm{mmr} .2015 .4368$

\begin{abstract}
The aim of the present study was to investigate the therapeutic efficacy of fibroblast growth factor 10 (FGF10) in the promotion of healing, survival and expression of mucin in corneal epithelial cells in a rabbit dry eye model. A total of 12 healthy female New Zealand white rabbits were divided randomly into three groups. The lacrimal glands were injected with saline either alone (normal control group) or with concanavalin A (Con A), with either topical phosphate-buffered saline (PBS; PBS control group) or $25 \mu \mathrm{g} / \mathrm{ml}$ FGF10 (FGF10 treatment group). Lacrimal gland inflammation, tear function, corneal epithelial cell integrity, cell apoptosis and mucin expression were subsequently assessed. Lacrimal gland tissue biopsies were performed in conjunction with histology and electron microscopy observations. Tear meniscus height (TMH) and tear meniscus area (TMA) were measured using Fourier domain-optical coherence tomography. Tear membrane break-up time (TBUT) was also assessed and corneal fluorescein staining was performed. The percentages of apoptotic corneal and conjunctival (Cj) epithelial cells (ECs) were counted using a terminal deoxynucleotidyl transferase-mediated dUTP nick end labeling method. The mRNA expression levels of Mucl were determined using reverse transcription-quantitative polymerase chain reaction analyses. The TMH and TMA values of the PBS and treatment groups were found to be significantly reduced, compared with those of the normal control group 3 days after Con A injection. However, the TMH and TMA of the FGF10 treatment group were higher, compared with those of the PBS group 3 and 7 days after treatment, respectively. Furthermore, the FGF10 treatment group exhibited prolonged TBUT, reduced corneal fluorescein staining and repaired epithelial cell ultrastructure7 days after treatment. The percentages of apoptotic
\end{abstract}

Correspondence to: Professor Bilian Ke, Department of Ophthalmology, Shanghai General Hospital, Shanghai Jiaotong University School of Medicine, 100 Haining Road, Shanghai 200080 , P.R. China

E-mail: kebilian@126.com

Key words: fibroblast growth factor 10, rabbit dry eye model, cornea, apoptosis, Mucl corneal- and $\mathrm{Cj}$-ECs in the FGF10 treatment group were significantly reduced, compared with those in the PBS group. FGF10 significantly induced the mRNA expression of Mucl in the corneal epithelial cells, compared with the normal control group, and induced higher mRNA expression levels of Muc1 in the $\mathrm{Cj}-\mathrm{ECs}$, compared with the PBS control group. In the present study, the rabbit dry eye model was successfully established 3 days after lacrimal gland Con A injection. FGF10 eye drops increased TMH and TMA, promoted corneal epithelial healing, reduced apoptosis of the corneal- and $\mathrm{Cj}$-ECs and led to increased expression of Muc1.

\section{Introduction}

Dry eye is a multifactorial disease involving a number of different pathological mechanisms, including instability of the tear film, tear hyperosmolarity and inflammation of the ocular surface $(1,2)$. Several US and international population-based studies have demonstrated that dry eye occurs in between 5 and $35 \%$ of the population, and is not confined to the elderly, but is also present in young individuals (3-5). The variety of dry eye symptoms commonly include stinging or burning of the eyes, scratchiness, viscous mucus in or around the eyes, excessive eye irritation resulting from smoke or wind and excess tearing, all of which can not only affect the visual quality and comfort of the patient, but in severe cases can result in corneal ulcers, loss of vision and other pathologies (6-10). At present, therapeutic agents including artificial tears are able to temporarily relieve dry eye, however, treatments that promote ocular surface healing, and increase mucin secretion and tear film stability are limited.

A previous in vitro study demonstrated that fibroblast growth factor 10 (FGF10) is associated with mucin production and the promotion of the proliferation of cultured rat conjunctival (Cj) epithelial cells (ECs) (11), which suggested that FGF10 may be useful in the treatment of dry eye. FGF10, a member of the fibroblast growth factor family, is also known as keratinocyte growth factor 2 and promotes the growth, proliferation and differentiation of epithelial cells $(12,13)$. It has also been reported to be an important growth factor in the regulation of corneal epithelial wound healing (14).

Mucins are important structural and functional components of the tear film and are critical for protection of the corneal and 
conjunctival epithelium $(15,16)$. Corneal damage and inflammation result in an increase in mucin production by the conjunctiva and induce the upregulation of FGF10. Although growth factors may promote the proliferation of $\mathrm{Cj}$-ECs and stimulate mucin production and secretion, previous studies have reported that the abnormal expression of conjunctival mucin is implicated in ocular surface disorders, including dry eye (17-19).

In the present in vivo study, the therapeutic efficacy of FGF10 was examined in an inflammation-induced rabbit dry eye model. In addition the effect of FGF10 was further evaluated on corneal epithelial cell healing, apoptosis and the expression of mucin.

\section{Materials and methods}

Animal protocols. A total of 12 New Zealand white female rabbits (2.0-2.5 kg; Laboratory Animal Center of Shanghai, Shanghai, China) were treated, according to the Association for Research in Vision and Ophthalmology Statement for the Use of Animals in Ophthalmic and Vision Research. The present study was approved by the ethics committee of the Shanghai General Hospital, Shanghai Jiaotong University School of Medicine (Shanghai, China). The animals were maintained in a controlled environment with a 12-h light/dark cycle at $18 \pm 3^{\circ} \mathrm{C}$ and a minimum of $30 \%$ humidity. The rabbits $(n=4$ per group) were randomly assigned to one of three groups. In the phosphate-buffered saline (PBS) control group, rabbit lacrimal glands were injected with the T-cell mitogen concanavalin A (Con A; Sigma-Aldrich, St. Louis, MO, USA) and PBS eye drops (Jieshikang Biotechnology Co., Ltd., Qingdao, China) were topically applied. In the FGF10 treatment group, rabbit lacrimal glands were injected with Con A and $25 \mu \mathrm{g} / \mathrm{ml} \mathrm{FGF10}$ eye drops (donated by Associate Professor Xiaojie Wang of the Wenzhou Eye Research Institute, Wenzhou, Zhejiang, China) were topically applied. In the normal control group, rabbit lacrimal glands were injected with saline and no eye drops were applied. All procedures were performed under anesthesia by subcutaneous administration of ketamine hydrochloride (30 mg/kg) and xylazine (6 mg/kg) (Jieshikang Biotechnology Co., Ltd.). The anesthetized animals were placed in a conventional holder and received injections into the right lacrimal glands. Injections were performed by retracting the lower eyelid and inserting the needle $\sim 1 \mathrm{~cm}$ from the nasal canthus into the suborbital space, to a depth of $\sim 6 \mathrm{~mm}$. A single $30 \mu \mathrm{l}$ volume of saline (normal control group) or Con A (PBS control and FGF10 treatment groups) was injected into the lacrimal gland using a 30-gauge needle and a Hamilton Gastight ${ }^{\circledR}$ syringe (Hamilton, Reno, NV, USA). The experimentally treated eyes (FGF10 treatment group; $\mathrm{n}=4$ ) received $25 \mu \mathrm{g} / \mathrm{ml} \mathrm{FGF10} \mathrm{in}$ PBS, as reported by Wang et al (14), whereas the control eyes (PBS control group; $\mathrm{n}=4$ ) received PBS alone. These solutions were applied topically four times per day 3 days after the injections. No eye drops were applied in the normal control group.

Histopathological evaluation. Microscopic examinations were performed on the paraffin-embedded (Puzheng Biotechnology Co. Ltd, Shanghai, China), hematoxylin and eosin (H\&E; Gefan Biological Technology Co., Ltd., Shanghai, China)-stained sections of the excised rabbit lacrimal glands following animal sacrifice by intravenous injection of air into the ears of the anesthetized ears of the rabbits. The lacrimal glands were fixed and preserved in neutral buffered formalin (Hubei Xingyinhe Chemical Co., Ltd., Hubei, China), and the fixed gland tissue was dehydrated through ascending ethanol concentrations, embedded in paraffin, cut into $4 \mu \mathrm{m}$ sections and stained with $\mathrm{H} \& \mathrm{E}$ for light microscopy (Digital Biological Microscope BX53; Olympus Corporation, Tokyo, Japan) examination.

Optical coherence tomography (OCT) lower tear meniscus parameter measurement. Images of the lower tear meniscus were imaged at the inferior cornea-lid scan junction, with a 6-mm-vertical x 2.8-mm-depth scan, using a Fourier Domain-optical coherence tomography (FD-OCT) system (RTVue; Optovue, Inc., Fremont, CA, USA), using the measurement techniques described previously (20). Briefly, the OCT images were exported for manual computer caliper measurement using ReVue RTVue software (version 4.0; Optovue, Inc). The lower tear meniscus height (TMH) was defined as the distance between the cornea-meniscus junction and the lower eyelid-meniscus junction. The lower tear meniscus area (TMA) was the area enclosed by the lower tear meniscus, cornea and lower eyelid. All measurements were performed by one experienced investigator in a blinded-manner, and were recorded for data analysis. TMH was classified as follows: ,$+<200 \mu \mathrm{m} ;++,<180 \mu \mathrm{m} ;+++,<160$. A TMH of +++ was characteristic of dry eye (Fig. 1).

Corneal fluorescein staining and tear breakup time (TBUT) assessment. TBUT was determined by applying moist sodium fluorescein test strips (Tianjing Jingming New Technological Development Co., Ltd., Tianjing, China) onto the rabbit conjunctiva capsule, following which the eyelids were opened and closed manually to distribute the fluorescein within the tear film. Under slit lamp (Topcon Corporation, Tokyo, Japan) observation, the eye was held open and the time until one or more black spots or streaks appeared in the precorneal tear film was recorded. TBUT was classified as follows: +, 10-20 sec; ++, 5-10 sec; +++, $<5 \mathrm{sec}$; A TBUT of +++ was characteristic of dry eye.

Transmission electron microscopy (TEM) examination. Samples for TEM were fixed in 2.5\% glutaraldehyde (Hubei Xingyinhe Chemical Co., Ltd.) in $0.1 \mathrm{~mol} / 1$ phosphate buffer (pH 7.4) and then post-fixed in $1 \%$ osmium acid (Hubei Xingyinhe Chemical Co., Ltd.). Subsequent to dehydration with an ascending alcohol series, the samples were embedded in epoxy resin (Epok812 ${ }^{\circledR}$; Ohkenshoji Co., Ltd., Tokyo, Japan). Small sections $\left(1 \mathrm{~mm}^{3}\right)$ were cut from the middle area of the cornea. The sections were subjected to double staining with lead acetate (Hubei Xingyinhe Chemical Co., Ltd.) and uranyl acetate (Hubei Xingyinhe Chemical Co., Ltd.) and were observed using a transmission electron microscope (H-7000; Hitachi, Ltd., Tokyo, Japan).

Terminal deoxynucleotidyl transferase-mediated dUTP nick end labeling (TUNEL) staining. The TUNEL technique was used to evaluate apoptosis in the corneal- and $\mathrm{Cj}$-ECs. Each paraffin-embedded section was deparaffinized and rehydrated. Proteinase K (20 mg/l; Hubei Xingyinhe Chemical Co., Ltd.) was applied for $30 \mathrm{~min}$ and endogenous peroxidase was quenched using 3\% hydrogen peroxide (Hubei Xingyinhe Chemical Co., Ltd.) for $10 \mathrm{~min}$. An Annexin V-fluorescein isothiocyanate/propidium iodide Apoptosis Detection kit 
(Nanjing Keygen Biotech Co., Ltd., Nanjing, China) was used. The terminal deoxynucleotidyl transferase reaction was performed for $1 \mathrm{~h}$ at $37^{\circ} \mathrm{C}$ prior to application of diaminobenzidine chromogen (Hubei Xingyinhe Chemical Co., Ltd.).

Reverse transcription-quantitative polymerase chain reaction $(R T-q P C R)$. Total RNA was isolated from epithelial cells using TRIzol (Invitrogen Life Technologies, Carlsbad, CA, USA), according to the manufacturer's instructions. The total RNA $(1 \mu \mathrm{g})$ was used for cDNA synthesis using a Reverse Transcription System (Takara Bio, Inc., Otsu, Japan), according to the manufacturer's instructions. The cDNA was amplified by qPCR using primers specific for rabbit Mucl in a thermal cycler (PCR Sprint; Thermo Hybaid, Franklin, MA, USA). The primer sequences of Muc1 were forward 5'-GTG CTGTCGTCACCTCTGCCC-3' and reverse 5'-TGAGACCGA CGGGCTGGTGG-3'. GAPDH served as the internal control and the primer sequences used were forward 5'-CTGCCG CCTGGAGAAAGC-3' and reverse 5'-AGACGACCTGGT CCTCGGTG-3'. A standard reaction was performed in a total volume of $50 \mu \mathrm{l}$, consisting of $4 \mu \mathrm{l}$ cDNA, $2 \mu \mathrm{l}$ each of the

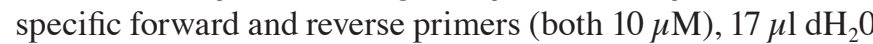
and $25 \mu 12$ X SYBR Green I PCR mix (Toyobo Co., Ltd., Osaka, Japan). The parameters used were as follows: $5 \mathrm{~min}$ at $94^{\circ} \mathrm{C}$, followed by 35 cycles of denaturation for $30 \mathrm{sec}$ at $94^{\circ} \mathrm{C}$, then extension for $1 \mathrm{~min}$ at $72^{\circ} \mathrm{C}$. The expression levels were quantified using the $2^{-\Delta \Delta \mathrm{CT}}$ method (21).

Statistical analysis. All data were statistically analyzed using SAS Software, version 8.2 (SAS Institute, Inc., Cary, NC, USA). Data were analyzed using a Student-Newman-Keuls test, following analysis of variance, which is the most widely used method for pairwise comparison $(22,23)$, to identify differences between the groups. The results are expressed as the mean \pm standard deviation. $\mathrm{P}<0.05$ was considered to indicate a statistically significant difference.

\section{Results}

Histopathological evaluation. Histological examination of the lacrimal glands $72 \mathrm{~h}$ following injection with $300 \mu \mathrm{g}$ Con A revealed moderately severe necrosis of the epithelial cells and inflammatory cell infiltration. By contrast, no evidence of inflammation or abnormality was observed following injection of $30 \mu \mathrm{l}$ saline (Fig. 2).

Lower tear meniscus measurement. The lower tear meniscus measurements, determined using FD-OCT are listed in Table I. The TMH and TMA measurements 3 days following Con A injection in the PBS and FGF10 groups were significantly reduced, compared with those of the saline-injected animals. At 3 days post-treatment, the TMH in the FGF10 group was significantly increased, compared with the PBS group. At 7 days post-treatment, the TMA of the FGF10 group was also increased.

Corneal fuorescein staining and TBUT. The baseline tear film stability in rabbits, measured as the TBUT, was $>30 \mathrm{sec}$ when assessed prior to lacrimal gland injection, and the TBUT of the normal control group rabbits injected with saline remained

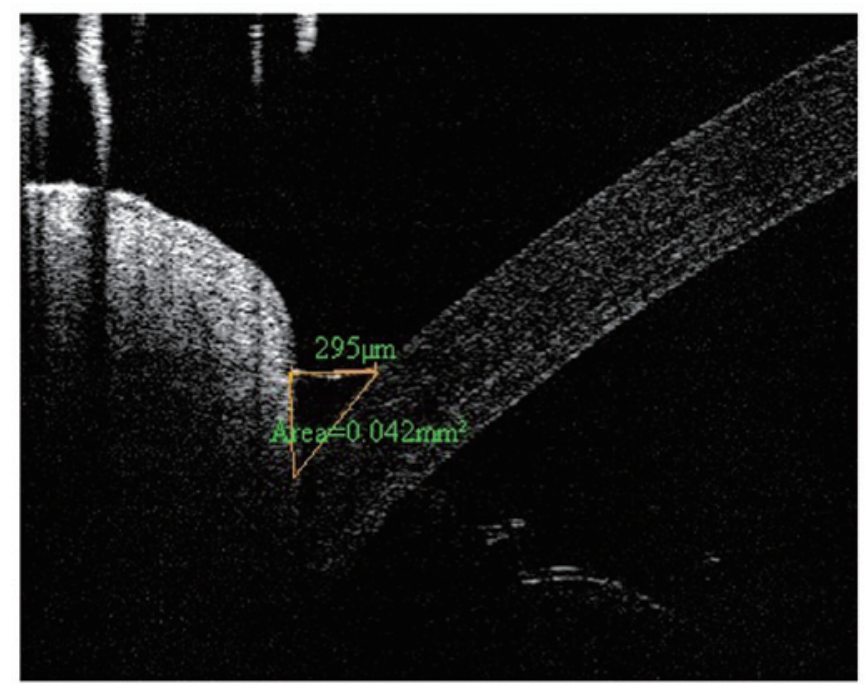

Figure 1. Fourier domain-optical coherence tomography tear meniscus scan. The height and area measurement of the lower tear meniscus is shown.

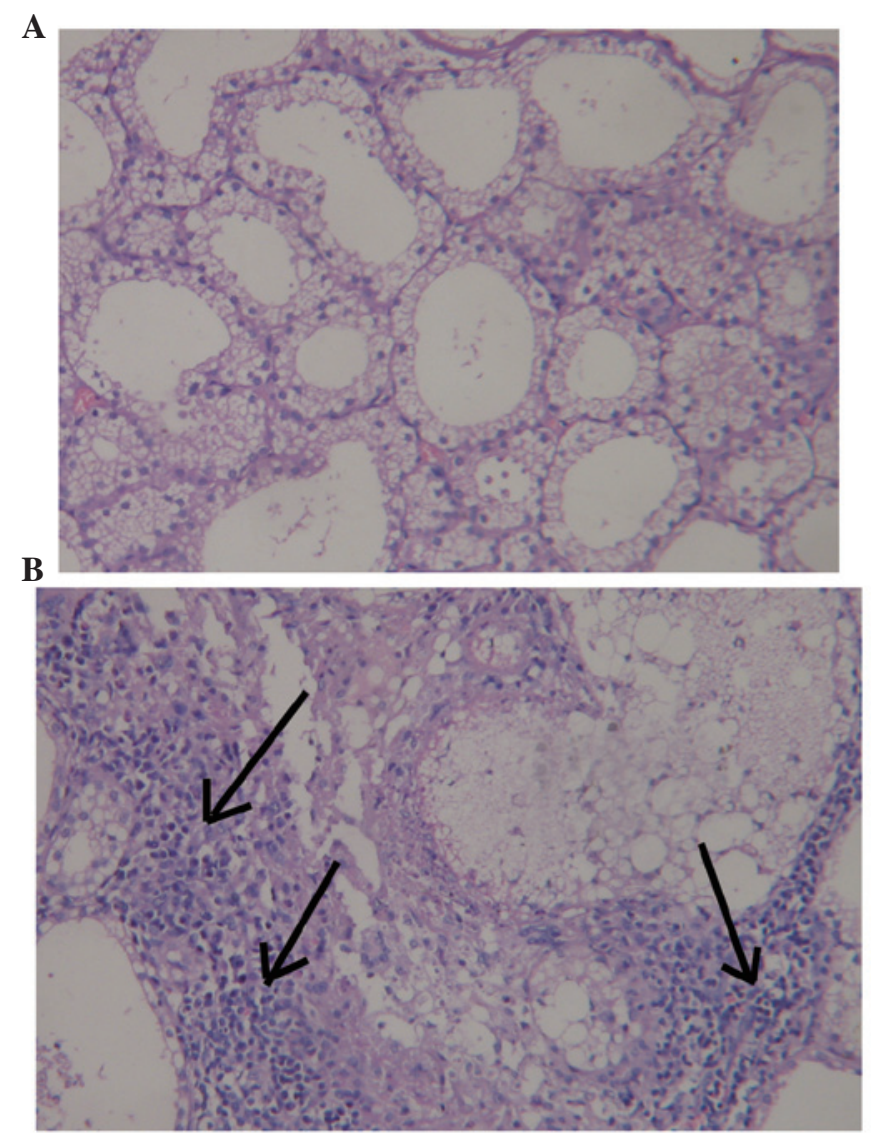

Figure 2. Histopathological evaluation of lacrimal glands following injection (magnification, x400). (A) No inflammation or abnormality was observed following saline injection. (B) Moderately severe necrosis of the epithelial cells and inflammatory cell infiltration (indicated by arrows) were observed following injection of concanavalin A.

unchanged for the duration of the investigation. By contrast, $300 \mu \mathrm{g}$ Con A resulted in a marked reduction in TBUT 3 days after lacrimal gland injection. However, TBUT increased 7 days after treatment in the FGF10 group (Table II). Punctate staining of fluorescein due to corneal epithelial injury signifi- 
Table I. Lower tear meniscus measurement.

\begin{tabular}{|c|c|c|c|c|c|c|}
\hline \multirow[b]{2}{*}{ Time-point } & \multicolumn{2}{|c|}{ PBS control group } & \multicolumn{2}{|c|}{ FGF10 treatment group } & \multicolumn{2}{|c|}{ Normal control group } \\
\hline & $\mathrm{TMH}(\mu \mathrm{m})$ & $\mathrm{TMA}\left(\mathrm{mm}^{2}\right)$ & TMH $(\mu \mathrm{m})$ & TMA $\left(\mathrm{mm}^{2}\right)$ & $\mathrm{TMH}(\mu \mathrm{m})$ & $\mathrm{TMA}\left(\mathrm{mm}^{2}\right)$ \\
\hline Day 0 & $231.36 \pm 11.59$ & $0.025 \pm 0.004$ & $224.06 \pm 7.81$ & $0.025 \pm 0.006$ & $221.75 \pm 5.85$ & $0.024 \pm 0.006$ \\
\hline 3 Days after injection & $155.47 \pm 14.57^{\mathrm{a}}$ & $0.011 \pm 0.005^{\mathrm{a}}$ & $151.11 \pm 37.90^{\mathrm{a}}$ & $0.009 \pm 0.005^{\mathrm{a}}$ & $227.00 \pm 17.39$ & $0.024 \pm 0.003$ \\
\hline 3 Days after treatment & $161.00 \pm 21.63^{\mathrm{a}}$ & $0.011 \pm 0.005^{\mathrm{a}}$ & $229.50 \pm 39.53^{\mathrm{b}}$ & $0.019 \pm 0.005$ & $244.25 \pm 28.59$ & $0.027 \pm 0.005$ \\
\hline 7 Days after treatment & $183.07 \pm 10.39^{a}$ & $0.014 \pm 0.002^{\mathrm{a}}$ & $254.00 \pm 16.97$ & $0.034 \pm 0.004^{b}$ & $230.50 \pm 16.26$ & $0.025 \pm 0.005$ \\
\hline
\end{tabular}

${ }^{a} \mathrm{P}<0.05$ vs. normal control group; ${ }^{\mathrm{b}} \mathrm{P}<0.05$ vs. PBS control group. PBS, phosphate-buffered saline; FGF10, fibroblast growth factor 10; TMH, tear meniscus height; TMA, tear meniscus area.

Table II. Tear membrane break-up time in the control and treatment groups.

\begin{tabular}{lccc}
\hline Time-point & PBS control group & FGF10 treatment group & Normal control group \\
\hline Day 0 & $>30 \mathrm{sec}$ & $>30 \mathrm{sec}$ & $>30 \mathrm{sec}$ \\
3 Days after injection & +++ & +++ & $>30 \mathrm{sec}$ \\
3 Days after treatment & ++ & ++ & $>30 \mathrm{sec}$ \\
7 Days after treatment & ++ & + & $>30 \mathrm{sec}$ \\
\hline
\end{tabular}

PBS, phosphate-buffered saline; FGF10, fibroblast growth factor 10. +, 10-20 sec; ++, 5-10 sec; +++, <5 sec.

Table III. Corneal and conjunctival epithelial cell apoptotic rates.

\begin{tabular}{llccr}
\hline Time-point & Tissue & PBS group (\%) & FGF10 group (\%) & Normal control (\%) \\
\hline 3 Days after treatment & Corneal & $28.3 \pm 1.36^{\mathrm{a}}$ & $12.8 \pm 1.15^{\mathrm{a}, \mathrm{b}}$ & $3.3 \pm 0.56$ \\
& Conjunctival & $41.7 \pm 0.63^{\mathrm{a}}$ & $34.0 \pm 1.22^{\mathrm{a}, \mathrm{b}}$ & $1.6 \pm 0.24$ \\
7 Days after treatment & Corneal & $61.3 \pm 5.29^{\mathrm{a}}$ & $44.9 \pm 1.37^{\mathrm{a}, \mathrm{b}}$ & $2.7 \pm 0.21$ \\
& Conjunctival & $42.0 \pm 2.02^{\mathrm{a}}$ & $29.8 \pm 1.06^{\mathrm{a}, \mathrm{b}}$ & $2.2 \pm 0.32$ \\
\hline
\end{tabular}

${ }^{\text {a }}<<0.05$ vs. normal control group; ${ }^{b} \mathrm{P}<0.05$ vs. PBS control group. PBS, phosphate-buffered saline; FGF10, fibroblast growth factor 10 .

cantly reduced in the FGF10 treatment group, compared with the PBS control group (Fig. 3).

TEM evaluation. TEM evaluation of the corneal epithelial cells following injection with $30 \mu \mathrm{l}$ saline, revealed no abnormalities. By contrast, injection with $300 \mu \mathrm{g}$ Con A led to epithelial cells, which exhibited a loss of microvilli structures and microfolds (Fig. 4), a widening of the intercellular space, shallow cellular edema, desmosome disintegration, mitochondrial swelling and cristae disappearance and ballooning degeneration, which is a form of cell death (Fig. 5A). At 3 days post-treatment, the FGF10 group exhibited light swelling of mitochondria and the appearance of cristae (Fig. 5B). At 7 days post-treatment, the epithelial cells of the FGF10 group exhibited microvilli structures and microfolds (Fig. 4) and also the appearance of mitochondrial cristae (Fig. 5C).

TUNEL staining evaluation. The corneal- and $\mathrm{Cj}$-EC apoptotic rates are listed in Table III. Compared with the normal control group, the apoptosis rate of the cells following injection with $300 \mu \mathrm{g}$ Con A was significantly higher $(\mathrm{P}<0.05)$. Subsequent to treatment for 3 and 7 days, the apoptotic rates in the FGF10 group were significantly reduced, compared with those in the PBS group ( $\mathrm{P}<0.05$; Figs. 6 and 7$)$.

RT-qPCR evaluation. The results of the RT-qPCR analysis demonstrated that the expression of Mucl was significantly upregulated by FGF10 treatment in the corneal epithelial cells, compared with the normal control group $(\mathrm{P}<0.05)$. FGF10 significantly upregulated corneal expression of Muc1, and decreased conjuctival expression of Muc1 (Fig. 8). Additionally, in the $\mathrm{Cj}$-ECs, Muc1 was significantly upregulated by FGF10, compared with the PBS control group ( $\mathrm{P}<0.05$; Fig. 8).

\section{Discussion}

The present in vivo study was performed using a short-term model of rabbit dry eye, which was strategically developed to 

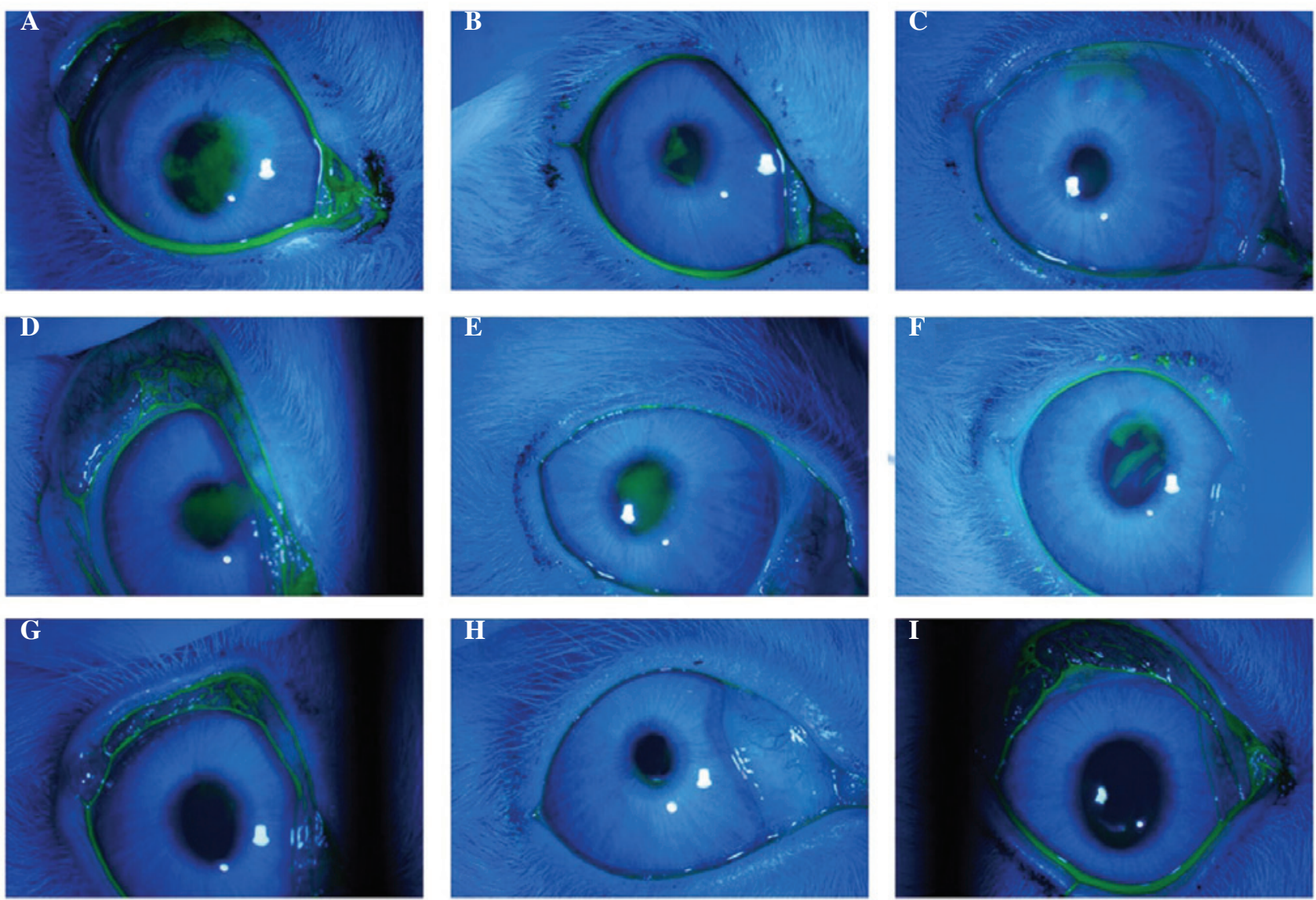

Figure 3. Corneal fluorescein staining of the three groups. FGF10 treatment group: (A) 3 days following Con A injection, clear fluorescein staining of the corneal epithelial injury was observed. (B) 3 days following FGF10 treatment, moderate fluorescein staining of the corneal epithelial injury remained. (C) 7 days following FGF10 treatment, light, punctate fluorescein staining of the corneal epithelial injury remained. PBS control group: (D) 3 days following Con A injection, clear fluorescein staining of the corneal epithelial injury was observed. (E) 3 days and (F) 7 days following PBS treatment, fluorescein staining of the corneal epithelial injury remained. In the normal control group $(\mathrm{G})$ three days following saline injection, $(\mathrm{H})$ three days following no eye drop treatment, and (I) seven days followin no eye drop treatment, no punctuate fluorescein staining of the cornea epithelium was observed. FGF10, fibroblast growth factor 10; Con A, concanavalin A.
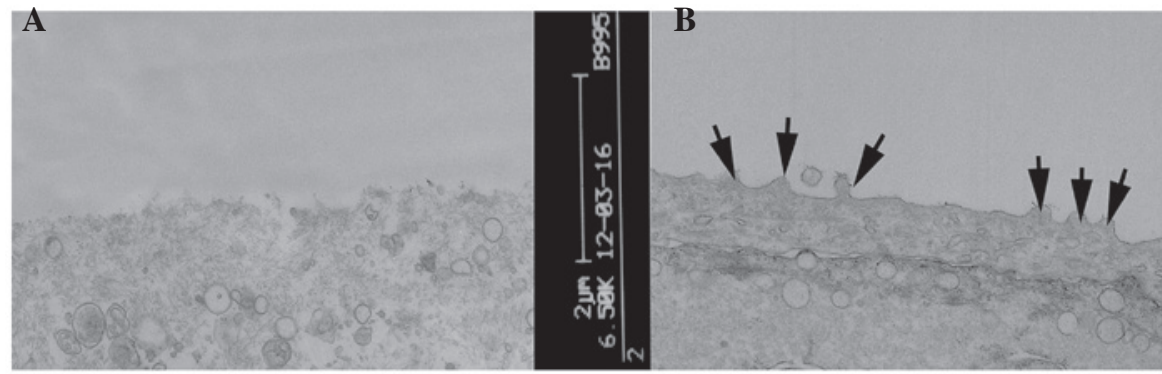

Figure 4. Corneal epithelial cells under an electron microscope. (A) Following injection with concanavalin A, the microvilli structures and microfolds were no longer visible. (B) 7 days following treatment with fibroblast growth factor 10, microvilli structure and microfolds were observed (indicated by the arrows).
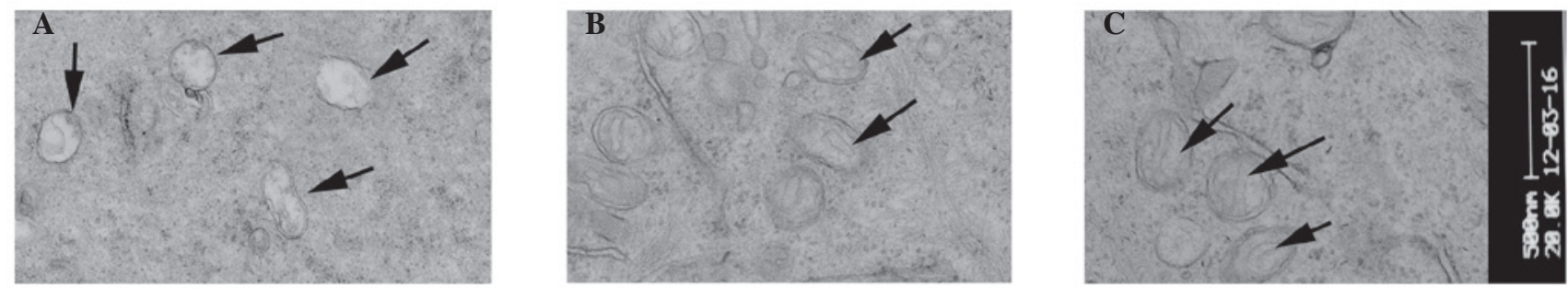

Figure 5. Corneal epithelial cells under an electron microscope. (A) Following injection with concanavalin A, the mitochondria of the cells were swollen, no cristae were identified and ballooning degeneration was evident. (B) 3 days following FGF10 treatment, light swelling of the mitochondria and cristae were visible (indicated by arrows). (C) 7 days following FGF10 treatment, light swelling of the mitochondria was visible (indicated by arrows). FGF10, fibroblast growth factor 10 . 

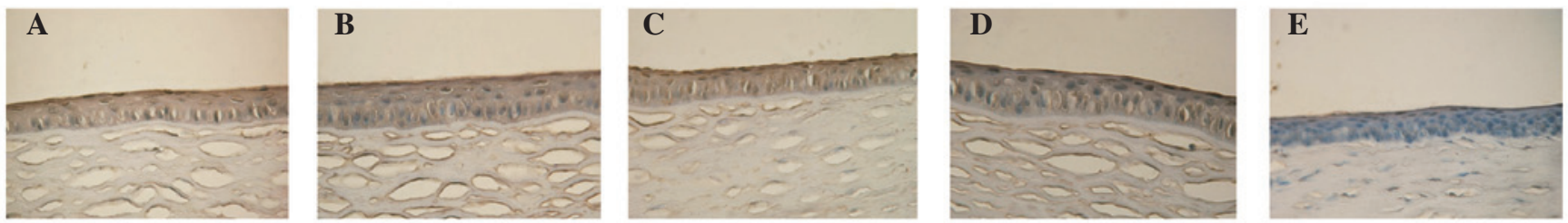

Figure 6. Terminal deoxynucleotidyl transferase-mediated dUTP nick end labeling of corneal cells (magnification, x250). Normal epithelial cells were stained intense blue and apoptotic epithelial cells were stained intense brown (diaminobenzidine). (A) PBS group and (B) FGF10 group 3 days after treatment. (C) PBS group, (D) FGF10 group and (E) normal control group 7 days after treatment. PBS, phosphate-buffered saline; FGF10, fibroblast growth factor 10.
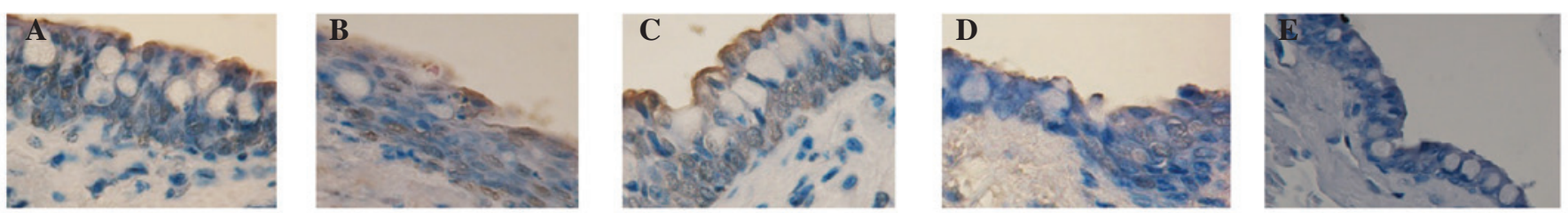

Figure 7. Terminal deoxynucleotidyl transferase-mediated dUTP nick end labeling of conjunctival epithelial cells (magnification, x250). Normal epithelial cells were stained intense blue and apoptotic epithelial cells were stained intense brown (diaminobenzidine). Conjunctiva in the (A) PBS group and (B) FGF10 group 3 days after treatment. Conjunctiva in the (C) PBS group, (D) FGF10 group and (E) normal control group 7 days after treatment. PBS, phosphate-buffered saline; FGF10, fibroblast growth factor 10.
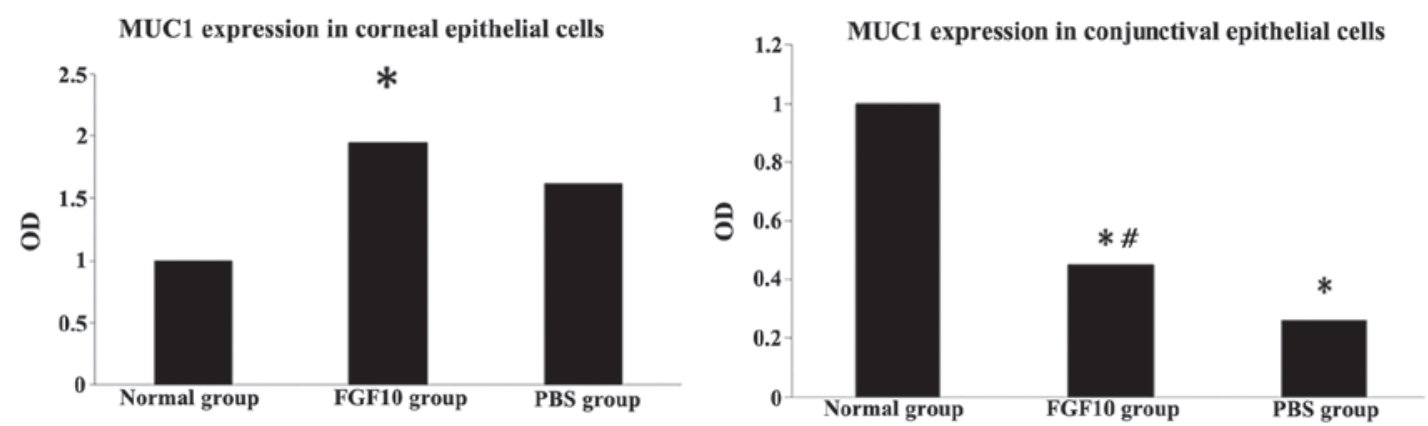

Figure 8. FGF10 increases corneal expression of Muc1 and decreases conjuctival expression of Muc1, compared with the untreated control. "P<0.05, vs. normal control group; ${ }^{\mathrm{P}} \mathrm{P}<0.05$, vs. PBS group. PBS, phosphate-buffered saline; FGF10, fibroblast growth factor 10; OD, optical density.

advance the discovery of drugs for dry eye (24). In the present study, the inflammatory response, characterized by necrosis of the epithelial cells of the lacrimal gland, was observed to be pronounced 3 days following Con A injection. Lacrimal gland inflammation significantly reduced the TMH, TMA, TBUT values, and increased corneal damage.

The measurement of lower tear meniscus using FD-OCT is an objective and noninvasive method of assessment $(25,26)$. Several previous studies have reported the use of FD-OCT in measuring human TMH and TMA, which correlate well with the symptoms of dry eye disease and Schirmer's test $(24,27)$. However, to the best of our knowledge, no previous studies have used this method in the rabbit dry eye model. In the present study, FD-OCT was found to be accurate for the quantification of tear volume.

The present study demonstrated that FGF10 promoted TMH and TMA, and increased TBUT length in the rabbit dry eye. These data suggested that FGF10 provided effective treatment in the rabbit model of dry eye. FGF10 is a member of the FGF family, which is involved in a wide variety of physiological and pathological processes, including inflammation, repair and regeneration $(13,28)$. Our previous in vitro study (11) demonstrated that FGF10 promoted the proliferation of rat Cj-ECs. The present study, demonstrated for the first time, to the best of our knowledge, that FGF10 can increase TMH and maintain tear film stability.

Wang et al (14) reported that FGF10 is important in successful corneal wound healing, which is consistent with the observations of the present study that FGF10 repaired corneal damage in the dry eye model. In the present study, TEM revealed that FGF10 induced the repair of the structure of microvilli, microfolds and mitochondria of epithelial cells, which may affect their apoptosis. Previous studies have reported that FGF10 attenuates DNA damage and apoptosis of epithelial cells, in part, by mitogen-activated protein kinase/extracellular signal-related kinase-dependent signaling, which affects the mitochondria-regulated death pathway $(29,30)$. Therefore, the apoptosis of corneal- and Cj-ECs was further investigated in the present study. The TUNEL staining assay demonstrated that the percentage of apoptotic cells in the FGF10 treatment group was significantly lower than that of the PBS control group. This suggested that FGF10 may act to protect corneal- and Cj-ECs from apoptosis in this rabbit dry eye model. 
Mucins are high-molecular weight glycoproteins, which are involved in the protection of the corneal and conjunctival epithelia. Our previous study (11) revealed that FGF10 stimulates the expression and production of mucin in rat $\mathrm{Cj}$-ECs. In the present study, it was observed in corneal epithelial cells that the expression of Muc1 was significantly upregulated by FGF10 treatment. In Cj-ECs, the normal control group exhibited the highest expression level of Mucl, which was higher than that of the FGF10 treatment group. This indicated that, in this short-term animal model of dry eye, conjunctival injury may be so severe in the Con A injection animals that the Cj-ECs were unable compensate for the lack of moisture. Caffery et al (31) reported that higher expression levels of Muc1 in patients with Sjogren's syndrome may represent compensatory or protective responses to chronic insult to the ocular surface. The expression levels of Muc1 in the conjunctiva may be complex and exhibit different expression levels over the course of the progression of dry eye. As Muc1 was significantly upregulated by FGF10 treatment in the present study, it was hypothesized that FGF10 promotes the expression of Mucl. A long-term follow-up investigation is, however, required in order to confirm this conclusion.

In conclusion, the data reported in the present study indicated that the rabbit inflammation-induced dry eye model may be successfully established within 3 days following Con A injection, and that FGF10 may exert the following effects in the rabbit dry eye model: Increased TMH and TMA, corneal epithelial healing, reduced apoptosis of the corneal- and $\mathrm{Cj}$-ECs and increased Mucl production. As the present study is the first, to the best of our knowledge, to examine the effect of FGF10 in the rabbit dry eye model, the biological function data associated with FGF10 suggested that FGF10 may be a promising candidate for the treatment of dry eye.

\section{Acknowledgements}

The present study was supported by the Natural Science Foundation of China (grant no. 81200713), the Natural Science Foundation of Shanghai, China (grant no. 09ZR1425400) and the Foundation of Shanghai Jiaotong University, Shanghai, China (grant no. YG2011MS62).

\section{References}

1. Lemp MA: Advances in understanding and managing dry eye disease. Am J Ophthalmol 146: 350-356, 2008.

2. The definition and classification of dry eye disease: Report of the definition and classification subcommittee of the international dry eye WorkShop (2007). Ocul Surf 5: 75-92, 2007.

3 . Friedman NJ: Impact of dry eye disease and treatment on quality of life. Curr Opin Ophthalmol 21: 310-316, 2010.

4. The epidemiology of dry eye disease: Report of the epidemiology subcommittee of the international dry eye WorkShop (2007). Ocul Surf 5: 93-107, 2007

5. Uchino M, Dogru M, Uchino Y, Fukagawa K, Shimmura S, Takebayashi T, Schaumberg DA and Tsubota K: Japan Ministry of Health study on prevalence of dry eye disease among Japanese high school students. Am J Ophthalmol 146: 925-929, 2008.

6. Guo B, Lu P, Chen X, Zhang W and Chen R: Prevalence of dry eye disease in Mongolians at high altitutde in China: The Henan eye study. Ophthalmic Epidemiol 17: 234-241, 2010.

7. Schein OD, Muñoz B, Tielsch JM, Bandeen-Roche K and West S: Prevalence of dry eye among the elderly. Am J Opthalmol 124: 723-728, 1997.

8. Brewitt H and Sistani F: Dry eye disease: The scale of the problem. Surv Ophthalmol 45 (Suppl2): S199-S202, 2001.
9. Baudouin C: The pathology of dry eye: Surv Ophthalmol 45 (Suppl2) S211-S220, 2001

10. Rolando $\mathbf{M}$ and Zierhut M: The ocular surface and tear film and their dysfunctionin dry eye disease. Surv Ophthalmol 45 (Supp12): S207-S210, 2001.

11. Ma MM, Zhang ZW, Niu W, Kelimu J, Ke B and Zhang Z: Fibroblast growth factor 10 upregulates the expression of mucins in rat conjunctival epithelial cells. Mol Vis 17: 2789-2797, 2011.

12. Marchese C, Felici A, Visco V, Lucania G, Igarashi M, Picardo M, Frati L and Torrisi MR: Fibroblast growth factor 10 induces proliferation and differentiation of human primary cultured keratinocytes. J Invest Dermatol 116: 623-628, 2001.

13. Jurjus A, Atiyeh BS, Abdallah IM, Jurjus RA, Hayek SN, Jaoude MA, Gerges A and Tohme RA: Pharmacological modulation of wound healing in experimental burns. Burns 33: 892-907, 2007.

14. Wang X, Zhou X, Ma J, Jiao Y, Zhang R, Huang Z, Xiao J, Zhao B, Qian H, Li X and Tian H: Effects of keratinocyte growth factor-2 on corneal epithelial wound healing in a rabbit model of carbon dioxide laser injury. Biol Pharm Bull 33: 971-976, 2010.

15. Gipson IK: The ocular surface: The challenge to enable and protect vision-the friendenwald lecture. Invest Ophthalmol Vis Sci 48: 4391-4398, 2007.

16. Gipson IK and Argüeso P: Role of mucins in the function of the corneal and conjunctival epithelia. Int Rev Cytol 231: 1-49, 2003.

17. Dartt DA: Control of mucin production by ocular surface epithelial cells. Exp Eye Res 78: 173-185, 2004.

18. Micera A, Stampachiacchiere B, Normando EM, Lambiase A, Bonini S and Bonini S: Nerve growth factor modulates toll-like receptor (TLR) 4 and 9 expression in cultured primary VKC conjunctival epithelial cells. Mol Vis 15: 2037-2044, 2009.

19. Gu J, Chen L, Shatos MA, Rios JD, Gulati A, Hodges RR and Dartt DA: Presence of EGF growth factor ligands and their effects on cultured rat conjunctival goblet cell proliferation. Exp Eye Res 86: 322-334, 2008.

20. Savini G, Barboni P and Zanini M: Tear meniscus evaluation by optical coherence tonmgraphy. Ophthalmic Surg Lasers Imaging 37: 112-118, 2006.

21. Livak KJ and Schmittgen TD: Analysis of relative gene expression data using real-time quantitative PCR and the 2(-Delta Delta C(T)) Method. Methods 25: 402-408, 2010.

22. Feinstein AR: Principle of Medical Statistics. Chapman and Hall/CRC, London, 2005.

23. Rosner B: Fundamentals of Biostatistics. 6th Edition. Brooks Cole, Pacific Grove, CA, 2005.

24. Nagelhout TJ, Gamache DA, Roberts L, Brady MT and Yanni JM: Preservation of tear film integrity and inhibition of corneal injury by dexamethasone in a rabbit model of lacrimal gland inflammation-induced dry eye. J Ocular Pharmacol Ther 21: 139-148, 2005.

25. Baikoff G, Lutun E, Ferraz C and Wei J: Analysis of the eye's anterior segment with optical coherence tomography. Static and dynamic study. J Fr Ophtalmol 28: 343-352, 2005 (In French).

26. Baikoff G, Lutun E, Ferraz C and Wei J: Static and dynamic analysis of the anterior segment with optical coherence tomography. J Cataract Refract Surg 9: 1843-1850, 2004.

27. Nguyen P, Huang D, Li Y, Sadda SR, Ramos S, Pappuru RR and Yiu SC: Correlation between optical coherence tomography-derived assessments of lower tear meniscus parameters and clinical features of dry eye disease. Cornea 31 : 680-685, 2012

28. Ohuchi H, Hori Y, Yamasaki M, Harada H, Sekine K, Kato S and Itoh N: FGF10 acts a major ligand for FGF receptor $2 \mathrm{IIIb}$ in mouse multi-organ development. Biochem Biophys Res Commum 277: 643-649, 2000.

29. Upadhyay D, Panduri V and Kamp DW: Fibroblast growth factor-10 prevents asbestos-induced alveolar epithelial cell apoptosis by a mitogen-activated protein kinase-dependent mechanism. Am J Respir Cell Mol Biol 32: 232-238, 2005.

30. Tai CC, Curtis JL, Sala FG, Del Moral PM, Chokshi N, Kanard RJ, Al Alam D, Wang J, Burns RC and Ford HR: Induction of fibroblast growth factor 10 (FGF10) in the ileal crypt epithelium after massive small bowel resection suggests a role for FGF10 in gut adaptation. Deve Dyn 238: 294-301, 2009.

31. Caffery B, Heynen ML, Joyce E, Jones L, Ritter R III and Senchyna M: MUC1 expression in Sjogren's syndrome, KCS and control subjects. Mol Vis 16: 1720-1727, 2010. 